\title{
Development of a Dielectric Barrier Discharge Ion Source for Ambient Mass Spectrometry
}

\author{
$\mathrm{Na} \mathrm{Na}$, Mengxia Zhao, Sichun Zhang, Chengdui Yang, \\ and Xinrong Zhang \\ Department of Chemistry, Key Laboratory for Atomic and Molecular Nanosciences of the Education \\ Ministry, Tsinghua University, Beijing, China
}

\begin{abstract}
A new ion source based on dielectric barrier discharge was developed as an alternative ionization source for ambient mass spectrometry. The dielectric barrier discharge ionization source, termed as DBDI herein, was composed of a copper sheet electrode, a discharge electrode, and a piece of glass slide in between as dielectric barrier as well as sample plate. Stable low-temperature plasma was formed between the tip of the discharge electrode and the surface of glass slide when an alternating voltage was applied between the electrodes. Analytes deposited on the surface of the glass slide were desorbed and ionized by the plasma and the ions were introduced to the mass spectrometer for mass analysis. The capability of this new ambient ion source was demonstrated with the analysis of 20 amino acids, which were deposited on the glass slide separately. Protonated molecular ions of $[\mathrm{M}+\mathrm{H}]^{+}$were observed for all the amino acids except for L-arginine. This ion source was also used for a rapid discrimination of L-valine, L-proline, L-serine and L-alanine from their mixture. The limit of detection was 3.5 pmol for L-alanine using single-ion-monitoring (SIM). Relative standard deviation (RSD) was $5.78 \%$ for $17.5 \mathrm{nmol}$ of L-alanine $(n=5)$. With the advantages of small size, simple configuration and ease operation at ambient conditions, the dielectric barrier discharge ion source would potentially be coupled to portable mass spectrometers. (J Am Soc Mass Spectrom 2007, 18, 1859-1862) (C) 2007 American Society for Mass Spectrometry
\end{abstract}

$\mathrm{V}$ arious desorption ionization (DI) sources have been developed, including laser desorption/ ionization (LDI) [1, 2], fast atom bombardment (FAB) $[3,4]$, and matrix-assisted laser desorption ionization (MALDI) $[5,6]$, etc. Recently, a new family of DI techniques has emerged for direct detection of samples on surfaces that allow ions to be generated under ambient conditions and then analyzed by MS, such as desorption electrospray ionization (DESI) or direct analysis in real time (DART) [7-11]. For DESI, sprayed charged droplets were directed at the ambient object of interest and release ions from the sample surface; for DART, the energetic agents generated by a gas discharge were used to desorb and ionize the analyte from samples. Despite these techniques being very elegant, the development of direct sampling and ambient ion sources with simple configurations are always desirable.

The design of a new ambient ionization source, termed as dielectric barrier discharge ionization (DBDI), is based on the concept of dielectric barrier discharge (DBD). The DBD is obtained at atmospheric pressure with a dielectric layer between two electrodes applied with alternating voltages $[12,13]$. The dielectric limits

Address reprint requests to Professor Xinrong Zhang, Department of Chemistry, Tsinghua University, Beijing 100084, P.R. China. E-mail: xrzhang@chem.tsinghua.edu.cn the average current density in the gas space, forming stable low-temperature plasma with large amount of high energetic electrons [14, 15]. The non-equilibrium plasma properties of DBD can be used to develop new ionization method. It is expected that the source based on DBD needs neither electrosprayed solvent to form desorbed ions as for DESI, nor a device with complex configuration as for DART. Reduced pressure is not required to maintain a stable discharge for DBDI compared with glow discharge ion sources [16-19].

In the present work, a DBDI source has been designed with advantages of small size and simple configuration for ambient desorption/ionization. Twenty amino acids deposited on a glass slide were analyzed individually to demonstrate the potentials of the present ion source.

\section{Experimental}

\section{Instrument}

DBD power supplies were purchased from Beili Guoke Co. Ltd. (Beijing, China). Ions were mass analyzed using a commercial linear ion trap mass spectrometer (Finnigan LTQ, Thermo Electron Co., San Jose, CA). Data were processed using the instrument software interface (Xcalibur version 1.4 SR1). Mass spectrometry 
conditions were as follows: source voltage (applied to the spray capillary), $0 \mathrm{kV}$; tube lens voltage, $95 \mathrm{~V}$; capillary temperature, $275^{\circ} \mathrm{C}$; heated capillary voltage, $33 \mathrm{~V}$; multipole rf amplitude $\left(\mathrm{V}_{p-p}\right), 400 \mathrm{~V}$; and multiplier voltages 1 and 2, $-1200 \mathrm{~V}$. The ion injection time was set to $50 \mathrm{~ms}$, and the number of microscans was set to one.

\section{Reagents}

All reagents were of analytical-reagent grade. Amino acids were purchased from Beijing Dingguo Biotechnology Co. Ltd. (Beijing, China). Helium (99.99\%) and argon (99.99\%) from Huayuan Gas (Beijing, China) were used as the carrier gases. Monosodium glutamate was the product of Henan Lotus Gourmet Powder Incorporate Ltd. (Henan, China). Water was deionized and further purified with a Milli-Q water purification system (Millipore, Milford, MA).

\section{Sample Preparation}

For each amino acid, a sample solution containing 40 nmol of the analyte was deposited on a piece of filter paper about $3 \mathrm{~mm} \times 3 \mathrm{~mm}$. Twenty pieces of filter paper containing the individual amino acids were placed on the glass slide to form a $4 \times 5$ array for analysis.

\section{Results and Discussion}

\section{Design of DBDI Source}

The schematic of the DBDI device is shown in Figure 1. A hollow stainless steel needle $(20 \mathrm{~mm}$ long, $0.2 \mathrm{~mm}$ i.d.) was used as a discharge electrode. Helium or other gases flowed through the needle at a speed of 12 to 48

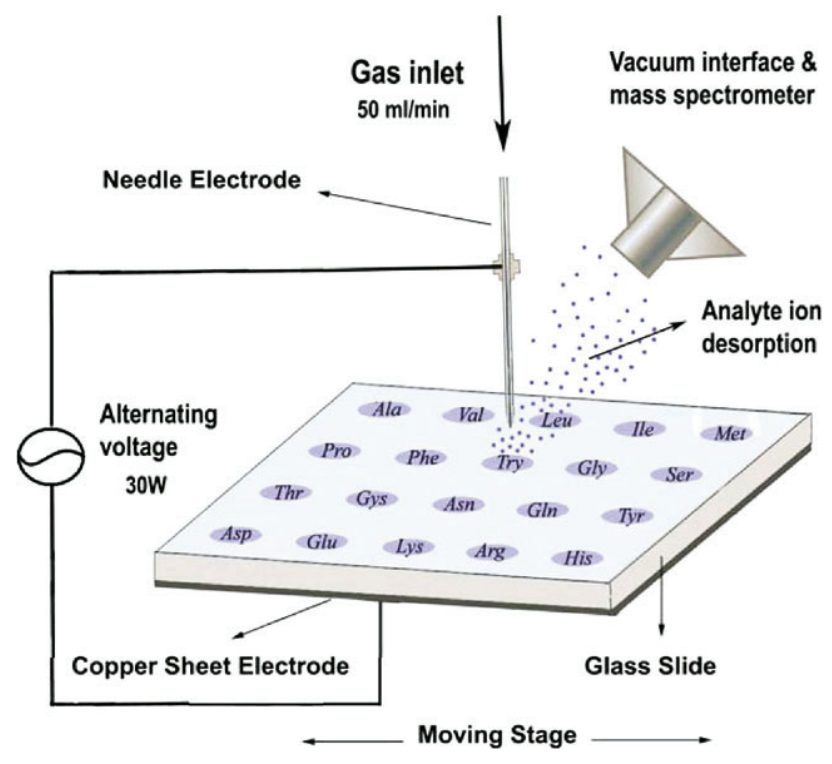

Figure 1. Schematic of the DBDI source. $\mathrm{m} / \mathrm{s}$. A copper sheet $(25 \mathrm{~mm} \times 75 \mathrm{~mm})$ was used as the counter electrode. A piece of glass slide $(25.4 \mathrm{~mm} \times 76.2$ $\mathrm{mm} \times 1.2 \mathrm{~mm}$ ) was inserted between two electrodes and mounted on the surface of the copper sheet. The glass slide served as both the discharge barrier and the sample plate. The distance between the needle electrode tip and the surface of glass slide was 5 to 10 $\mathrm{mm}$. The glass slide and copper sheet were mounted on a $3 \mathrm{D}$ moving stage, allowing them to be positioned at any chosen point with respect to the needle electrode.

An alternating voltage of 3500 to $4500 \mathrm{~V}$ with a frequency of $20.3 \mathrm{kHz}$ was applied between two electrodes, forming stable plasma between the tip of the needle electrode and glass slide. The analytes on the surface of glass slide were desorbed and ionized by the plasma. Then the produced ions were introduced to the mass spectrometer for mass analysis.

\section{Desorption and Ionization of Amino Acids}

The base peaks of $[\mathrm{M}+\mathrm{H}]^{+}$were observed from all the amino acids except for $\mathrm{L}$-arginine. The ions of [M + $\mathrm{H}-\mathrm{HCOOH}]^{+},\left[\mathrm{M}+\mathrm{H}-\mathrm{H}_{2} \mathrm{O}\right]^{+}$, or $\left[\mathrm{M}+\mathrm{H}-\mathrm{NH}_{3}\right]^{+}$ were also observed, which were confirmed by collisioninduced dissociation (CID). Taking L-glutamic acid $\left(M_{r}=147\right)$ as an example, ions of $m / z 148\left([\mathrm{M}+\mathrm{H}]^{+}\right)$, $130\left(\left[\mathrm{M}+\mathrm{H}-\mathrm{H}_{2} \mathrm{O}\right]^{+}\right), 102\left([\mathrm{M}+\mathrm{H}-\mathrm{HCOOH}]^{+}\right)$and $84\left(\left[\mathrm{M}+\mathrm{H}-\mathrm{HCOOH}-\mathrm{H}_{2} \mathrm{O}\right]^{+}\right)$were obtained. Detail information of 20 amino acids is included in Supporting Information, which can be found in the electronic version of the article (Table S1). The observed fragmentation patterns were consistent with the reported fragmentation mechanisms for amino acids [19, 20]. For L-arginine, the absence of $[\mathrm{M}+\mathrm{H}]^{+}$is possibly due to the excessive fragmentation since the energy of metastable helium or fast electrons is much larger than the ionization potential of L-arginine [18].

The DBDI source could also be used for the fast discrimination of amino acids in a mixture without pre-separation. Figure 2 shows the mass spectrum obtained from the mixture of L-valine, L-proline, L-serine, and L-alanine. The protonated molecular ion of each amino acid $(m / z 118,116,106$, and 90) was obtained.

The spectra of L-leucine and L-isoleucine were sufficiently different for their discrimination. Lisoleucine produced the ion of $m / z 132\left([\mathrm{M}+\mathrm{H}]^{+}, 100 \%\right)$ and $m / z 86\left([\mathrm{M}+\mathrm{H}-\mathrm{HCOOH}]^{+}, 45 \%\right)$. For Lleucine, ions of $\mathrm{m} / \mathrm{z} 132$ (100\%), 128 (31\%), 86 (91\%), and $84(29 \%)$ were observed. The ion of $\mathrm{m} / \mathrm{z} 128$ was present as the ion of $\left[\left(\mathrm{CH}_{3}\right)_{2} \mathrm{CCHC}(\mathrm{COOH}) \mathrm{NH}_{2}\right]^{+}$ with stable conjugated $\mathrm{C}=\mathrm{C}$ bonds. Moreover, the ion of $m / z 84$ was generated with the loss of $\mathrm{HCOOH}$ from $\left[\left(\mathrm{CH}_{3}\right)_{2} \mathrm{CCHC}(\mathrm{COOH}) \mathrm{NH}_{2}\right]^{+}$. The peak assignment of L-leucine had been verified by CID.

\section{Characteristics of Ionization Process}

Both protonated molecular ions and characteristic fragment ions can be generated during the discharge pro- 


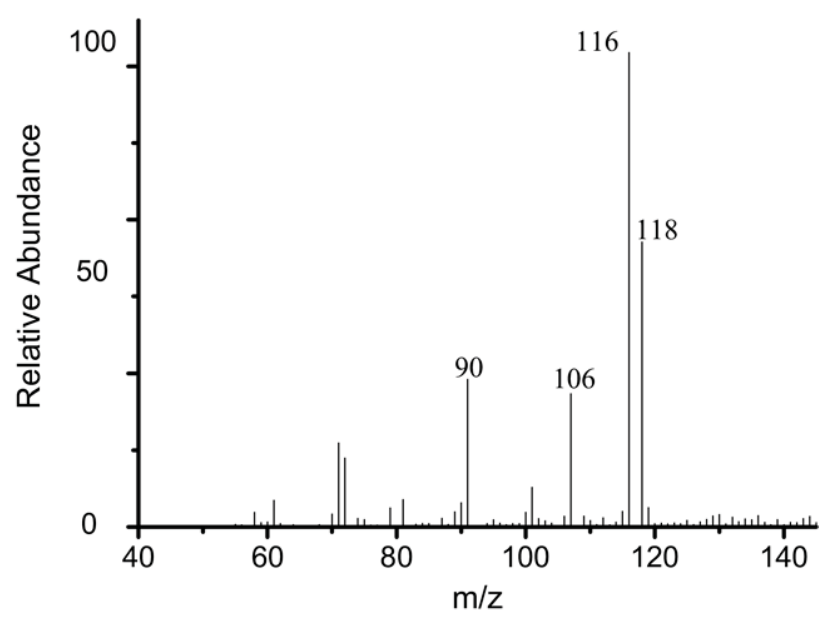

Figure 2. Mass spectrum of the mixture of L-valine $\left(M_{r} 117\right)$, L-proline $\left(M_{r} 115\right)$, L-serine $\left(M_{r} 105\right)$, and L-alanine $\left(M_{r} 89\right)$. The amino acids were diluted by water, and the amount of each amino acid was $40 \mathrm{nmol}$ in the mixture solution.

cess. It was observed that the intensity ratios of the fragment ions to protonated molecular ions increase with time. As illustrated by the mass spectrum of L-glutamic acid, protonated molecular ion $(\mathrm{m} / \mathrm{z} 148$, $100 \%$ ) was the most abundant ion at $3 \mathrm{~s}$ after the discharge started (Figure 3a). It became 19 and $9 \%$ at $6 \mathrm{~s}$ and $9 \mathrm{~s}$, respectively (Figure $3 \mathrm{~b}$ and $\mathrm{b}$ ). The relative intensity of the protonated molecular ion $(\mathrm{m} / \mathrm{z} 148)$ decreased (Figure 3e) with time, while the relative intensity of fragment ion ( $/ z / z 130)$ increased (Figure 3f).
Thus, it was important to control the discharge time to obtain protonated ions. However, the characteristic time dependence of the fragmentation on the ionization process was potentially useful for getting fragment ions. In our experiments, a total spectral acquisition time of $3 \mathrm{~s}$ was selected to avoid fragmentation. (Details are shown in the Supplementary Material section, which can be found in the electronic version of this article.)

\section{The Ionization Mechanism in DBDI}

The ionization process is affected by the polarity of discharge gas, the proton affinity, and ionization potential of analytes, which is complicated enough to involve several mechanisms, such as the bombardment of fast atoms, electron impact, and electron transfer. In addition, the reaction with water in air and Penning ionization by the bombardment of metastable gas may occur [21-23]. The fragment ions may be generated by the collision of the ions with electrons or metastable species in the plasma. Furthermore, the thermal effects in the gas phase may also lead to the fragmentation. Since the process is very complicated, further studies are required for the mechanism of ionization by DBDI.

\section{Optimizations of Ionization Parameters}

Optimization of the ionization parameters for the detection of amino acids was carried out by varying the

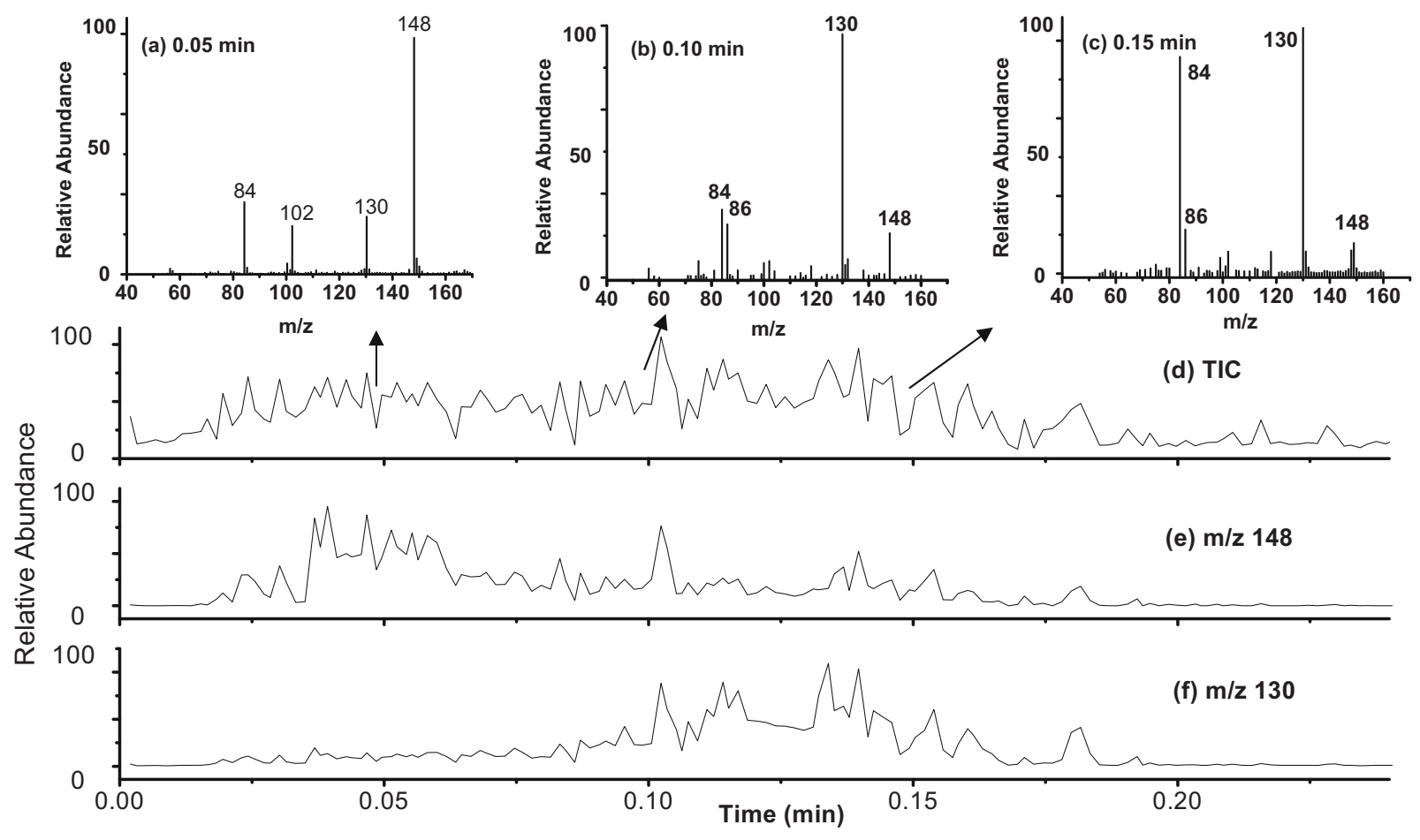

Figure 3. Desorption and ionization of L-glutamic acid at different discharge time; (a) $0.05 \mathrm{~min}$, (b) $0.10 \mathrm{~min}$, (c) $0.15 \mathrm{~min}$. The TIC of L-glutamic acid (d), intensities of ion $\mathrm{m} / \mathrm{z} 148$ (e), and ion $\mathrm{m} / \mathrm{z} 130(\mathbf{f})$ 
discharge power, the kind of gas, the gas speed, and the dielectric material.

The signal intensity of assignment ions was enhanced with the increase of the discharge power from 5 to $30 \mathrm{~W}$ (Figure S-1). Further increase of the power caused the fragmentation of the molecular ions, which was consistent with previous reports [16, 24]. A power supply of $30 \mathrm{~W}$ was used in the experiments.

Various gases, including helium, argon, nitrogen, and air were tested for generating the DBD plasma to desorb/ionize analytes. The mass spectra obtained with different gases were shown in Figure S-2. The highest signal/noise ratio was obtained with helium gas, while relatively good results were also achieved with other gases. The mass spectra recorded at different gas speed of helium were shown in Figure S-3. A gas speed of 12 $\mathrm{m} / \mathrm{s}$ was used, which was about 20 to 30 times lower than that of other ambient ion sources [7, 11].

The analytes can be desorbed and ionized from various solid surfaces, including glass slide, filter paper, plastic, or glass slide eroded by hydrofluoric acid. It is interesting that the mass spectra of amino acids can be obtained on TLC plate, which indicates that the DBDI could be used for the identification of compounds after sample separation by TLC plate.

\section{Analytical Performance and the Sample Analysis}

The DBDI ion source has been shown to have good reproducibility for the detection of amino acids. The peak $m / z 90$ of L-alanine was monitored for a test of the reproducibility. The RSD was $5.78 \%$ for five times desorption of L-alanine at the amount of $17.5 \mathrm{nmol}$ on each spot. The limit of detection (LOD) was 3.5 pmol for L-alanine using single-ion-monitoring (SIM). The results indicated that DBDI method had the comparable sensitivity to the existing methods $[25,26]$.

The analysis of a commercial monosodium glutamate was carried out and L-glutamic acid $\left(M_{r} 147\right)$ in the condiment was identified based on the characteristic ions of $m / z 148,130,102$, and 84 . The mass spectra of L-glutamic acid and the commercial monosodium glutamate are shown in Figure S-4.

\section{Conclusions}

A dielectric barrier discharge ion source has been developed to desorb and ionize the analytes directly from surface under ambient conditions. This simple and small ion source is suitable for portable mass spectrometers. The application of DBDI for higher molecular weight and more complicated species will be investigated. The present ion source can potentially be used for imaging of biological samples since the needle-plate discharge could be localized within an area of several micrometers.

\section{Acknowledgments}

The authors acknowledge financial support by the National Natural Science Foundation of China (grants 20635002, 20535020, and 20575034).

\section{References}

1. Antonov, V. S.; Letokhov, V. S.; Matveyets, Y. A.; Shibanov, A. N. Sputtering of Neutral Molecules and Molecular Ions from the Adenine Crystal Surface Induced by the UV Picosecond Laser Pulse. Laser Chem. $1982,1,37-43$.

2. Karas, M.; Bachmann, D.; Hillenkamp, F. Influence of the Wavelength in High-Irradiance Ultraviolet Laser Desorption Mass Spectrometry of Organic Molecules. Anal. Chem. 1985, 57, 2935-2939.

3. Macfarlane, R. D.; Torgerson, D. F. Califorium-252 Plasma Desorption Mass Spectroscopy. Science 1976, 191, 920-925.

4. Barber, M.; Bordoli, R. S.; Sedgwick, R. D.; Tyler, A. N. Fast Atom Bombardment of Solids (FAB): A New Ion Source for Mass Spectrometry. JCS Chem. Comm. 1981, 325-327.

5. Laiko, V. V.; Baldwin, M. A.; Burlingame, A. L. Atmospheric Pressure Matrix-Assisted Laser Desorption/Ionization Mass Spectrometry. Anal. Chem. 2000, 72, 652-657.

6. O'Connor, P. B.; Costello, C. E. A High Pressure Matrix-Assisted Laser Desorption/Ionization Fourier Transform Mass Spectrometry Ion Source for Thermal Stabilization of Labile Biomolecules. Rapid Commun. Mass Spectrom. 2001, 15, 1862-1868

7. Cooks, R. G.; Ouyang, Z.; Takats, Z.; Wiseman, J. M. Ambient Mass Spectrometry. Science 2006, 311, 1566-1570.

8. Yang, P.; Cooks, R. G.; Ouyang, Z. Gentle Protein Ionization Assisted by High-Velocity Gas Flow. Anal. Chem. 2005, 77, 6174-6183.

9. Wiseman, J. M.; Puolitaival, S. M.; Takáts, Z.; Cooks, R. G.; Caprioli, R. M. Mass Spectrometric Profiling of Intact Biological Tissue by Using Desorption Electrospray. Angew. Chem. Int. Ed. 2005, 44, 7094-7097.

10. Cody, R. B.; Laramée, J. A.; Durst, H. D. Versatile New Ion Source for the Analysis of Materials in Open Air Under Ambient Conditions. Anal. Chem. 2005, 77, 2297-2302.

11. Cody, R. B.; Laramée, J. A.; Nilles, J. M.; Durst, H. D. Direct Analysis in Real Time (DART) Mass Spectrometry. JEOL News 2005, 40, 8-12.

12. Sonnenfeld, A.; Tun, T. M.; Zajičková, L.; Kozlov, K. V.; Wagner, H. E. Behnke, J. F.; Hippler, R. Deposition Process Based on Organosilicon Precursors in Dielectric Barrier Discharges at Atmospheric Pressure-A Comparison. Plasma Polym. 2001, 6, 237-266.

13. Ling, Y. M. Probe Diagnosis of Electron Temperature and Electron Energy Distribution in Low-pressure Dielectric Barrier Discharge. Phys. Plasma 2005, 12, 113504-1-113504-6.

14. Kogelschatz, U. Dielectric-Barrier Discharges: Their History, Discharge Physics, and Industrial Applications. Plasma Chem. Plasma P 2003, 23, $1-46$.

15. Lee, D.; Park, J. M.; Hong, S. H.; Kim, Y. Numerical Simulation on Mode Transition of Atmospheric Dielectric Barrier Discharge in HeliumOxygen Mixture. IEEE Trans. Plasma Sci. 2005, 33, 949-957.

16. McLuckey, S. A.; Glish, G. L.; Asano, K. G.; Grant, B. C. Atmospheric Sampling Glow Discharge Ionization Source for the Determination of Trace Organic Compounds in Ambient Air. Anal. Chem. 1988, 60, 2220-2227.

17. Newman, K.; Mason, R. S. Organotin Speciation Using Fast Flow Glow Discharge Mass Spectrometry. J. Anal. At. Spectrom. 2005, 20, 830-838.

18. Berkout, V. D. Fragmentation of Protonated Peptide Ions via Interaction with Metastable Atoms. Anal. Chem. 2006, 78, 3055-3061.

19. Klassen, J. S.; Kebarle, P. Collision-Induced Dissociation Threshold Energies of Protonated Glycine, Glycinamide, and Some Related Small Peptides and Peptide Amino Amides. J. Am. Chem. Soc. 1997, 119, 6552-6563.

20. O'Hair, R. A.; Broughton, P. S.; Styles, M. L.; Frink, B. T.; Hadad, C. M. The Fragmentation Pathways of Protonated Cycline: A Computational Study. J. Am. Soc. Mass Spectrom. 2000, 11, 687-696.

21. Tsuchiya, M.; Kuwabara, H. Liquid Ionization Mass Spectrometry of Nonvolatile Organic Compounds. Anal. Chem. 1984, 56, 14-19.

22. Carazzato, D.; Bertrand, M. J. Characterization of a Glow Discharge Ion Source for the Mass Spectrometric Analysis of Organic Compounds. J. Am. Soc. Mass Spectrom. 1994, 5, 305-315.

23. Hiraoka, K.; Fujimaki, S.; Kambara, S.; Furuya, H.; Okazaki, S. Atmospheric-Pressure Penning Ionization Mass Spectrometry. Rapid Commum. Mass Spectrom. 2004, 18, 2323-2330.

24. Evans, E. H.; Pretorius, W.; Ebdon, L.; Rowland, S. Low-Pressure Inductively Coupled Plasma Ion Source for Molecular and Atomic Mass Spectrometry. Anal. Chem. 1994, 66, 3400-3407.

25. Langrock, T.; Czihal, P.; Hoffmann, R. Amino Acid Analysis by Hydrophilic Interaction Chromatography Coupled On-line to Electrospray Ionization Mass Spectrometry. Amino Acids 2006, 30, 291-297.

26. Yang, W. C.; Mirzaei, H.; Liu, X.; Regnier, F. E. Enhancement of Amino Acid Detection and Quantification by Electrospray Ionization Mass Spectrometry. Anal. Chem. 2006, 78, 4702-4747. 